\title{
The [a,b]-domination and [a,b]-total Domination of Graphs
}

\author{
Xiujun Zhang ${ }^{1,2}$, Zehui Shao ${ }^{1,2}$, Hong Yang ${ }^{1,2}$ \\ ${ }^{1}$ School of Information Science and Engineering, Chengdu University, Chengdu, China \\ ${ }^{2}$ Key Laboratory of Pattern Recognition and Intelligent Information Processing, Institutions of Higher Education of \\ Sichuan Province, Chengdu University, China \\ Correspondence: Zehui Shao, School of Information Science and Engineering, Chengdu University, Chengdu, 610106, \\ China. Tel: 86-135-518-62078. E-mail: zshao@cdu.edu.cn
}

Received: February 6, 2017 Accepted: May 10, 2017 Online Published: May 18, 2017

doi:10.5539/jmr.v9n3p38

URL: https://doi.org/10.5539/jmr.v9n3p38

\begin{abstract}
A subset $S$ of the vertices of $G=(V, E)$ is an $[a, b]$-set if for every vertex $v$ not in $S$ we have the number of neighbors of $v$ in $S$ is between $a$ and $b$ for non-negative integers $a$ and $b$, that is, every vertex $v$ not in $S$ is adjacent to at least $a$ but not more than $b$ vertices in $S$. The minimum cardinality of an $[a, b]$-set of $G$ is called the $[a, b]$-domination number of $G$. The $[a, b]$-domination problem is to determine the $[a, b]$-domination number of a graph. In this paper, we show that the [2,b]-domination problem is NP-complete for $b$ at least 3, and the [1,2]-total domination problem is NP-complete. We also determine the [1,2]-total domination and [1,2] domination numbers of toroidal grids with three rows and four rows.
\end{abstract}

Keywords: [a,b]-domination, [a,b]-total domination, NP-complete, toroidal grids

\section{Introduction}

Let $G$ be a graph, $S \subseteq V(G), v \in V(G)$. The open neighborhood of $v$ in $S,\{u \mid u v \in E(G), u \in S\}$, is denoted by $N_{S}(v)$. We write $N_{S}[v]=\{v\} \cup N_{S}(v)$. If $S=V(G)$, we write $N_{S}(v)=N(v)$ and $N_{S}[v]=N[v]$. Let $T \subseteq V(G)$, we write $N_{G}(T)=\cup_{x \in T} N_{G}(x)$. We omit the subscript $G$, that is to say, $N_{G}(T)=N(T)$. The diameter of $G$ is the maximum distance between the vertices of $G$, denoted by $\operatorname{diam}(G)$. The maximum degree of graph $G$ is denoted by $\Delta(G)$. For more definitions and notations of graph theory, please consult (Bondy \& Murty,1976). If $n \equiv r(\bmod p)$, we write $n \in \mathbb{Z}_{p}^{r}$.

The Cartesian product $F \square G$ of graphs $F$ and $G$ is a graph with the vertex set $F \times G$, and $(f, g)\left(f^{\prime}, g^{\prime}\right) \in E(F \square G)$ if either $f f^{\prime} \in E(F)$ and $g=g^{\prime}$, or $g g^{\prime} \in E(G)$ and $f=f^{\prime}$. For more information on the Cartesian product of graphs please consult(Hammack, Imrich \& Klavžar,2011). The graph $C_{m} \square C_{n}$ is called a toroidal grid with $m$ rows and $n$ columns, and it is denoted by $T_{m, n}$.

Recently, a variation of the domination problem, called [a,b]-set, was proposed and studied (Chellali, Haynes, Hedetniemi \& McRae, 2013; Yang \& Wu, 2014; Goharshady, Hooshmandasl \& Meybodi,2016). A vertex subset $S$ of a graph $G=(V, E)$ is an [a, b]-set if, $a \leq|N(v) \cap S| \leq b$ for every vertex $v \in V \backslash S$, that is, each vertex $v \in V \backslash S$ is adjacent to either one or two vertices in $S$. A vertex subset $S^{\prime}$ of a graph $G=(V, E)$ is an [a, b]-total set if, $a \leq\left|N(v) \cap S^{\prime}\right| \leq b$ for every vertex $v \in V$, that is, each vertex $v \in V$ is adjacent to either one or two vertices in $S^{\prime}$. The minimum cardinality of an [a, b]-set (resp. [a, b]-total set) of $G$, denoted by $\gamma_{[a, b]}(G)\left(\gamma_{[a, b]}^{t}(G)\right)$, is called the [a, b]- domination (resp. [a, b]-total domination) number of $G$. The [a, b]-domination (resp. [a, b]-total domination) problem is to determine the [a, b]- domination (resp. [a, b]-total domination) number of a graph. (Chellali et al., 2013) studied graph $G$ with $\gamma_{[1,2]}(G)=n$ and grid graphs and prove that the [1,2]-domination graph is NP-complete for bipartite graphs by transforming an instance of EXACT-3-COVER to the [1,2]-domination problem.

In this paper, we show that the [2,b]-domination problem is NP-complete for $b \geq 3$, and the [1,2]-total domination problem is NP-complete. We also determine the [1,2]-total domination and [1,2] domination numbers of toroidal grids $T_{3, n}$ and $T_{4, n}$.

\section{Complexity of the [a,b]-domination Problem}

In (Fiala, Golovach, Kratochvíl, Lidický \& Paulusma, 2012), the NP-completeness of Monotone Not-All-Equal p-Satisfiability was applied, and in this paper, we will reduce from the Not-All-Equal p-Satisfiability problem, which is also NP-complete for $p \geq 3$ (Schaefer, 1978).

The Not-All-Equal p-Satisfiability:

Instance: A collection $C=\left\{C_{1}, C_{2}, \cdots, C_{m}\right\}$ of clauses on a finite set $X=\left\{x_{1}, x_{2}, \cdots, x_{n}\right\}$ of boolean variables such that $\left|C_{i}\right|=p$ for $1 \leq i \leq m$. 
Question: Can the literals be assigned value true or false so that each clause has at least one true and at least one false variable?

\section{The [a,b]-domination problem:}

Instance: A graph $G$, and an integer $k$.

Question: Is there an [a,b]-dominating set of $G$ with weight at most $k$ ?

The [1,2]-total domination problem:

Instance: A graph $G$, and an integer $k$.

Question: Is there a [1,2]-total dominating set of $G$ with weight at most $k$ ?

We will prove the following result:

Theorem 1 The [2,b]-domination problem is NP-complete for $b \geq 3$.

Proof. Given a Not-All-Equal p-Satisfiability with $p=b$ instance $C$, we will construct a graph $G$ whose order is polynomially bounded in the size of $C$ such that $C$ is satisfiable if and only if $G$ has a [2,b]-set of size at most $k$.

we construct a polynomial transformation from an instance of the The Not-All-Equal p-Satisfiability to an instance of the [2,b]-domination problem. Let $I$ be an arbitrary instance of The Not-All-Equal $p$-Satisfiability with $p=b$ for the set of clauses $C=\left\{C_{1}, C_{2}, \cdots, C_{m}\right\}$ on the set of boolean variables $X=\left\{x_{1}, x_{2}, \cdots, x_{n}\right\}$. For every boolean variable $x$ occurring in $C$ we introduce a gadget $G_{x}$, where $V\left(G_{x}\right)=\{x, \bar{x}, a, b, c, y, z\}$ and $E\left(G_{x}\right)=\{x \bar{x}, x a, x b, x c, \bar{x} a, \bar{x} b, \bar{x} c, y a, y b, y c, z a, z b, z c$, $y z, x y, \bar{x} z\}$. For each clause $C$, we construct a clause gadget $G_{C}$ with specific vertex $w$, where $V\left(G_{C}\right)=\{w, d, e, f, g, h\}$ and $E\left(G_{C}\right)=\{w d, d e, d f, d g, h e, h f, h g\}$. If the literal $x$ occurs in clause $C$ we connect the specified vertex $x$ in $G_{x}$ with the vertex $w$.

Let $G$ denote the resulting graph. It can be seen that $C$ is satisfiable (in the sense of Not-All-Equal p-Satisfiability) if and only if $G$ has a [2,b]-set of size at most $2 n+2 m$. Moreover, we have that $G$ has $6 m+7 n$ vertices and $16 n+(p+7) m$ edges, and so it is a polynomial transformation. This completes the proof.

Theorem 2 The [1,2]-total domination problem is NP-complete.

Proof. Given Not-all-equal 3SAT instance $C$, we will construct a graph $G$ whose order is polynomially bounded in the size of $C$ such that $C$ is satisfiable if and only if $G$ has a [1,2]-total set of size at most $k$.

we construct a polynomial transformation from an instance of the Not-all-equal 3SAT problem to an instance of the [1,2]total domination problem. Let $I$ be an arbitrary instance of Not-all-equal 3SAT for the set of clauses $C=\left\{C_{1}, C_{2}, \cdots, C_{m}\right\}$ on the set of boolean variables $X=\left\{x_{1}, x_{2}, \cdots, x_{n}\right\}$. For every boolean variable $x$ occurring in $C$ we introduce a copy $G_{x}$ of the gadget $K$ which contains two specific vertices $x$ and $\bar{x}$, where $V(K)=\{x, \bar{x}, y, z, w, c\}, E(K)=\{x y, \bar{x} y, c x, c \bar{x}, c z, c w\}$. For each clause $C_{i}$, we construct a vertex labeled $c_{i}$. If the literal $x$ occurs in clause $C_{i}$ we connect the specified vertex $x$ in $G_{x}$ with the vertex $c_{i}$. (For an example see Figure. 1 where $C=\{x \vee y \vee z, x \vee \bar{z} \vee \bar{w}\}$ ).

Let $G$ denote the resulting graph. It can be seen that $C$ is satisfiable if and only if $G$ has a [1,2]-total set of size at most $2 n$. Moreover, we have that $G$ has $m+6 n$ vertices and $6 n+3 m$ edges, and so it is a polynomial transformation. This completes the proof.

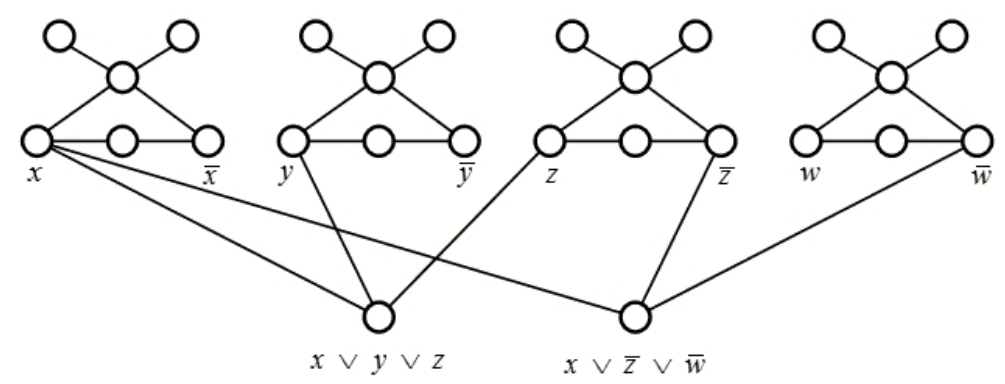

Figure 1. The graph $G$ for $C=\{x \vee y \vee z, x \vee \bar{z} \vee \bar{w}\}$ 


\section{The [1,2]-total Domination Number of Some Cartesian Product of Two Cycles}

We will use the following Observation.

Observation 1 Let $n \geq 3$ and $D$ is a [1,2]-total set of $T_{m, n}$. If $\left|D \cap C^{i}\right|=0$ for each $i \in\{j, j+2\}$ for some $j \in\{1,2, \cdots, n\}$. Then we have $\left|D \cap C^{j+1}\right| \geq\left\lceil\frac{m}{2}\right\rceil$.

Theorem 3 Let $n \geq 3$. Then $\gamma_{[1,2]}^{t}\left(T_{3, n}\right)=\left\lceil\frac{4 n}{5}\right\rceil$.

Proof. The lower bound can be found in (Thiagarajan \& Bhaskaram,2015), and we only need to show the upper bound. Let

$$
P=\left[\begin{array}{lllll}
0 & 1 & 0 & 0 & 0 \\
0 & 0 & 0 & 1 & 1 \\
0 & 1 & 0 & 0 & 0
\end{array}\right], Q=\left[\begin{array}{l}
0 \\
1 \\
0
\end{array}\right]
$$

Then the pattern $P^{k}$ induces a [1,2]-total set of with weight $4 k$ of $T_{3,5 k}$ for any $k \geq 1$, and the pattern $P^{k} Q^{i}$ induces a [1,2]-total set of with weight $4 k+i$ of $T_{3,5 k+i}$ for any $k \geq 1$ and $i \in\{1,2,3,4\}$. Thus, all the upper bounds are settled.

Theorem 4 Let $n \geq 4$. Then

$$
\gamma_{[1,2]}^{t}\left(T_{4, n}\right)= \begin{cases}n, & n \equiv 0(\bmod 4) \\ n+1, & n \equiv 1,3(\bmod 4), \\ n+2, & n \equiv 2(\bmod 4) .\end{cases}
$$

Proof. The lower bound can be found in (Thiagarajan \& Bhaskaram,2015), and we only need to show the upper bound. Let

$$
P=\left[\begin{array}{llll}
0 & 0 & 1 & 1 \\
0 & 0 & 0 & 0 \\
1 & 1 & 0 & 0 \\
0 & 0 & 0 & 0
\end{array}\right], Q_{1}=\left[\begin{array}{l}
1 \\
0 \\
1 \\
0
\end{array}\right], Q_{3}=\left[\begin{array}{ccc}
0 & 1 & 1 \\
0 & 0 & 0 \\
1 & 1 & 0 \\
0 & 0 & 0
\end{array}\right],
$$

then the pattern $P^{k}$ induces a [1,2]-total set of with weight $4 k$ of $T_{4,4 k}$ for any $k \geq 1$, the pattern $P^{k} Q_{1}^{2}$ induces a [1,2]-total set of with weight $4 k+4$ of $T_{4,4 k+2}$ for any $k \geq 1$, and the pattern $P^{k} Q_{i}$ induces a [1,2]-total set of with weight $4 k+i+1$ of $T_{4,4 k+i}$ for any $k \geq 1$ and $i \in\{1,3\}$. Thus, all the upper bounds are settled.

Theorem 5 Let $n \geq 6$. Then $\gamma_{[1,2]}^{t}\left(T_{5, n}\right) \leq\left\lceil\frac{9 n}{7}\right\rceil$.

Proof. We consider the following seven cases.

Case 1: $n \in \mathbb{Z}_{7}^{0}$.

Let $n=7 k$ and

$$
P=\left[\begin{array}{lllllll}
0 & 0 & 1 & 0 & 0 & 1 & 0 \\
1 & 0 & 0 & 0 & 0 & 0 & 0 \\
1 & 0 & 0 & 1 & 1 & 0 & 0 \\
1 & 0 & 0 & 0 & 0 & 0 & 0 \\
0 & 0 & 1 & 0 & 0 & 1 & 0
\end{array}\right]
$$

then the pattern $P^{k}$ induces a [1,2]-total set of with weight $9 k$ of $T_{5,7 k}$ for any $k \geq 1$.

Case 2: $n \in \mathbb{Z}_{7}^{1}$.

Let $n=7 k+1$

$$
P_{8}=\left[\begin{array}{llllllll}
0 & 0 & 0 & 1 & 1 & 0 & 0 & 0 \\
1 & 0 & 0 & 0 & 0 & 0 & 1 & 1 \\
0 & 0 & 1 & 1 & 0 & 0 & 0 & 0 \\
0 & 0 & 0 & 0 & 0 & 1 & 1 & 0 \\
1 & 1 & 0 & 0 & 0 & 0 & 0 & 0
\end{array}\right], P_{15}=\left[\begin{array}{lllllllllllllll}
1 & 0 & 0 & 0 & 0 & 0 & 0 & 1 & 1 & 0 & 0 & 0 & 0 & 0 & 1 \\
0 & 0 & 0 & 0 & 1 & 1 & 0 & 0 & 0 & 0 & 0 & 1 & 1 & 0 & 0 \\
0 & 1 & 1 & 0 & 0 & 0 & 0 & 0 & 1 & 1 & 0 & 0 & 0 & 0 & 0 \\
0 & 0 & 0 & 0 & 0 & 1 & 1 & 0 & 0 & 0 & 0 & 0 & 0 & 1 & 1 \\
0 & 0 & 1 & 1 & 0 & 0 & 0 & 0 & 0 & 0 & 1 & 1 & 0 & 0 & 0
\end{array}\right]
$$

and

$$
P_{22}=\left[\begin{array}{llllllllllllllllllllll}
0 & 0 & 1 & 0 & 0 & 1 & 0 & 0 & 1 & 0 & 0 & 1 & 0 & 0 & 1 & 0 & 0 & 0 & 1 & 0 & 0 & 1 \\
0 & 0 & 1 & 0 & 0 & 1 & 0 & 0 & 1 & 0 & 0 & 1 & 0 & 0 & 1 & 0 & 0 & 0 & 1 & 0 & 0 & 1 \\
0 & 0 & 0 & 0 & 0 & 0 & 0 & 0 & 0 & 0 & 0 & 0 & 0 & 0 & 0 & 0 & 1 & 0 & 0 & 0 & 0 & 0 \\
1 & 1 & 0 & 1 & 1 & 0 & 1 & 1 & 0 & 1 & 1 & 0 & 1 & 1 & 0 & 0 & 1 & 0 & 0 & 1 & 1 & 0 \\
0 & 0 & 0 & 0 & 0 & 0 & 0 & 0 & 0 & 0 & 0 & 0 & 0 & 0 & 0 & 0 & 1 & 0 & 0 & 0 & 0 & 0
\end{array}\right],
$$

then the pattern $P_{i}$ induces a [1,2]-total set of with the desired weight of $T_{5, i}$ for $i \in\{8,15,22\}$. 
Let

$$
Q_{22}=\left[\begin{array}{llllllllllllllllllllll}
0 & 0 & 1 & 0 & 0 & 1 & 0 & 0 & 1 & 0 & 0 & 1 & 0 & 0 & 1 & 0 & 0 & 1 & 0 & 0 & 1 & 0 \\
1 & 0 & 0 & 0 & 0 & 0 & 0 & 0 & 0 & 0 & 0 & 0 & 0 & 0 & 0 & 0 & 0 & 0 & 0 & 0 & 0 & 0 \\
1 & 0 & 0 & 1 & 1 & 0 & 1 & 1 & 0 & 1 & 1 & 0 & 1 & 1 & 0 & 1 & 1 & 0 & 1 & 1 & 0 & 0 \\
1 & 0 & 0 & 0 & 0 & 0 & 0 & 0 & 0 & 0 & 0 & 0 & 0 & 0 & 0 & 0 & 0 & 0 & 0 & 0 & 0 & 0 \\
0 & 0 & 1 & 0 & 0 & 1 & 0 & 0 & 1 & 0 & 0 & 1 & 0 & 0 & 1 & 0 & 0 & 1 & 0 & 0 & 1 & 0
\end{array}\right],
$$

then the pattern $P^{k} Q_{22}$ induces a [1,2]-total set of with weight $9 k+2$ of $T_{5,7 k+1}$ for any $k \geq 4$.

Case 3: $n \in \mathbb{Z}_{7}^{2}$.

Let $n=7 k+2$

$$
P_{9}=\left[\begin{array}{lllllllll}
0 & 1 & 1 & 0 & 1 & 1 & 0 & 1 & 1 \\
0 & 0 & 0 & 0 & 0 & 0 & 0 & 0 & 0 \\
1 & 0 & 0 & 1 & 0 & 0 & 1 & 0 & 0 \\
1 & 0 & 0 & 1 & 0 & 0 & 1 & 0 & 0 \\
0 & 0 & 0 & 0 & 0 & 0 & 0 & 0 & 0
\end{array}\right], P_{16}=\left[\begin{array}{llllllllllllllll}
0 & 1 & 0 & 0 & 1 & 0 & 0 & 0 & 1 & 0 & 0 & 1 & 0 & 0 & 1 & 0 \\
0 & 1 & 0 & 0 & 1 & 0 & 0 & 0 & 1 & 0 & 0 & 1 & 0 & 0 & 1 & 0 \\
0 & 0 & 0 & 0 & 0 & 0 & 1 & 0 & 0 & 0 & 0 & 0 & 0 & 0 & 0 & 0 \\
1 & 0 & 1 & 1 & 0 & 0 & 1 & 0 & 0 & 1 & 1 & 0 & 1 & 1 & 0 & 1 \\
0 & 0 & 0 & 0 & 0 & 0 & 1 & 0 & 0 & 0 & 0 & 0 & 0 & 0 & 0 & 0
\end{array}\right],
$$

then the patterns $P_{9}$ and $P_{16}$ induce a [1,2]-total set of with the desired weight of $T_{5,9}$ and $T_{5,16}$, respectively.

Let

$$
Q_{16}=\left[\begin{array}{cccccccccccccccc}
0 & 0 & 1 & 0 & 0 & 1 & 0 & 0 & 1 & 0 & 0 & 1 & 0 & 0 & 1 & 0 \\
1 & 0 & 0 & 0 & 0 & 0 & 0 & 0 & 0 & 0 & 0 & 0 & 0 & 0 & 0 & 0 \\
1 & 0 & 0 & 1 & 1 & 0 & 1 & 1 & 0 & 1 & 1 & 0 & 1 & 1 & 0 & 0 \\
1 & 0 & 0 & 0 & 0 & 0 & 0 & 0 & 0 & 0 & 0 & 0 & 0 & 0 & 0 & 0 \\
0 & 0 & 1 & 0 & 0 & 1 & 0 & 0 & 1 & 0 & 0 & 1 & 0 & 0 & 1 & 0
\end{array}\right]
$$

then the pattern $P^{k} Q_{16}$ induces a [1,2]-total set of with weight $9 k+3$ of $T_{5,7 k+2}$ for any $k \geq 3$.

Case 4: $n \in \mathbb{Z}_{7}^{3}$.

Let $n=7 k+3$

$$
P_{10}=\left[\begin{array}{cccccccccc}
1 & 0 & 0 & 0 & 0 & 0 & 0 & 0 & 0 & 0 \\
1 & 0 & 0 & 1 & 1 & 0 & 1 & 1 & 0 & 0 \\
1 & 0 & 0 & 0 & 0 & 0 & 0 & 0 & 0 & 0 \\
0 & 0 & 1 & 0 & 0 & 1 & 0 & 0 & 1 & 0 \\
0 & 0 & 1 & 0 & 0 & 1 & 0 & 0 & 1 & 0
\end{array}\right]
$$

then the pattern $P_{10}$ induces a [1,2]-total set of with the desired weight of $T_{5,10}$.

Let

$$
Q_{10}=\left[\begin{array}{cccccccccc}
0 & 0 & 1 & 0 & 0 & 1 & 0 & 0 & 1 & 0 \\
1 & 0 & 0 & 0 & 0 & 0 & 0 & 0 & 0 & 0 \\
1 & 0 & 0 & 1 & 1 & 0 & 1 & 1 & 0 & 0 \\
1 & 0 & 0 & 0 & 0 & 0 & 0 & 0 & 0 & 0 \\
0 & 0 & 1 & 0 & 0 & 1 & 0 & 0 & 1 & 0
\end{array}\right],
$$

then the pattern $P^{k} Q_{10}$ induces a [1,2]-total set of with weight $9 k+4$ of $T_{5,7 k+3}$ for any $k \geq 2$.

Case 5: $n \in \mathbb{Z}_{7}^{4}$.

Let $n=7 k+4$

$$
Q_{4}=\left[\begin{array}{cccc}
0 & 0 & 1 & 0 \\
1 & 0 & 1 & 0 \\
1 & 0 & 0 & 0 \\
1 & 0 & 0 & 0 \\
0 & 0 & 1 & 0
\end{array}\right]
$$

then the pattern $P^{k} Q_{4}$ induces a [1,2]-total set of with weight $9 k+6$ of $T_{5,7 k+4}$ for any $k \geq 2$.

Case 6: $n \in \mathbb{Z}_{7}^{5}$.

Let $n=7 k+5$

$$
P_{12}=\left[\begin{array}{llllllllllll}
0 & 0 & 0 & 0 & 0 & 0 & 0 & 0 & 0 & 0 & 0 & 0 \\
0 & 1 & 1 & 0 & 1 & 1 & 0 & 1 & 1 & 0 & 1 & 1 \\
0 & 0 & 0 & 0 & 0 & 0 & 0 & 0 & 0 & 0 & 0 & 0 \\
1 & 0 & 0 & 1 & 0 & 0 & 1 & 0 & 0 & 1 & 0 & 0 \\
1 & 0 & 0 & 1 & 0 & 0 & 1 & 0 & 0 & 1 & 0 & 0
\end{array}\right],
$$




$$
P_{19}=\left[\begin{array}{lllllllllllllllllll}
1 & 0 & 0 & 1 & 0 & 0 & 1 & 1 & 0 & 1 & 1 & 0 & 1 & 1 & 0 & 1 & 1 & 0 & 1 \\
0 & 0 & 0 & 1 & 0 & 0 & 0 & 0 & 0 & 0 & 0 & 0 & 0 & 0 & 0 & 0 & 0 & 0 & 0 \\
0 & 1 & 0 & 0 & 0 & 1 & 0 & 0 & 1 & 0 & 0 & 1 & 0 & 0 & 1 & 0 & 0 & 1 & 0 \\
0 & 1 & 0 & 0 & 0 & 1 & 0 & 0 & 1 & 0 & 0 & 1 & 0 & 0 & 1 & 0 & 0 & 1 & 0 \\
0 & 0 & 0 & 1 & 0 & 0 & 0 & 0 & 0 & 0 & 0 & 0 & 0 & 0 & 0 & 0 & 0 & 0 & 0
\end{array}\right],
$$

then the patterns $P_{12}$ and $P_{19}$ induce a [1,2]-total set of with the desired weight of $T_{5,12}$ and $T_{5,19}$, respectively.

Let

$$
Q_{19}=\left[\begin{array}{lllllllllllllllllll}
0 & 0 & 1 & 0 & 0 & 1 & 0 & 0 & 1 & 0 & 0 & 1 & 0 & 0 & 1 & 0 & 0 & 1 & 0 \\
1 & 0 & 0 & 0 & 0 & 0 & 0 & 0 & 0 & 0 & 0 & 0 & 0 & 0 & 0 & 0 & 0 & 0 & 0 \\
1 & 0 & 0 & 1 & 1 & 0 & 1 & 1 & 0 & 1 & 1 & 0 & 1 & 1 & 0 & 1 & 1 & 0 & 0 \\
1 & 0 & 0 & 0 & 0 & 0 & 0 & 0 & 0 & 0 & 0 & 0 & 0 & 0 & 0 & 0 & 0 & 0 & 0 \\
0 & 0 & 1 & 0 & 0 & 1 & 0 & 0 & 1 & 0 & 0 & 1 & 0 & 0 & 1 & 0 & 0 & 1 & 0
\end{array}\right],
$$

then the pattern $P^{k} Q_{19}$ induces a [1,2]-total set of with weight $9 k+7$ of $T_{5,7 k+5}$ for any $k \geq 4$.

Case 7: $n \in \mathbb{Z}_{7}^{5}$.

Let $n=7 k+6$

$$
Q_{6}=\left[\begin{array}{llllll}
0 & 1 & 0 & 0 & 1 & 0 \\
1 & 0 & 0 & 0 & 0 & 0 \\
1 & 0 & 1 & 1 & 0 & 0 \\
1 & 0 & 0 & 0 & 0 & 0 \\
0 & 1 & 0 & 0 & 1 & 0
\end{array}\right]
$$

then the pattern $P^{k} Q_{6}$ induces a [1,2]-total set of with weight $9 k+8$ of $T_{5,7 k+6}$ for any $k \geq 2$.

Therefore, all the upper bounds are obtained.

4. Some Graphs $G$ with $\gamma(G)=\gamma_{[1,2]}(G)$

In 2013, Chellali et al. proposed the following Question (see [(Chellali et al., 2013), Question 8]):

Question 1 For which graphs is $\gamma(G)=\gamma_{[1,2]}(G)$ ?

The following results concerning Question 1 are established in (Chellali et al., 2013):

Theorem 6 If $G$ is claw-free, then $\gamma(G)=i(G)=\gamma_{[1,2]}(G)$.

Corollary 1 If a graph $G$ has maximum degree $\Delta(G) \leq 2$, then $\gamma(G)=\gamma_{[1,2]}(G)$.

Corollary 2 For paths $G=P_{n}$ or cycles $G=C_{n}$, then $\gamma(G)=\gamma_{[1,2]}(G)$.

Proposition 1 If every vertex $v$ of a graph $G$ of order $n \geq 2$ is either a support vertex or has degree at most 2, then $\gamma(G)=\gamma_{[1,2]}(G)$.

Corollary 3 If $T$ is a caterpillar, then $\gamma(T)=\gamma_{[1,2]}(T)$.

Let the corona $G \circ K_{1}$ be the graph obtained from a graph $G=(V, E)$ by attaching a leaf to each vertex $v \in V$. Then Corollary 4 If $G$ is a corona $H \circ K_{1}$, then $\gamma(G)=\gamma_{[1,2]}(G)$.

Proposition 2 If $G$ is a non-trivial graph with $\Delta(G) \geq|V(G)|-3$, then $\gamma(G)=\gamma_{[1,2]}(G)$.

Theorem 7 If $G$ is $P_{4}$-free, then $\gamma(G)=i(G)=\gamma_{[1,2]}(G)$.

The toroidal grid is neither a claw-free graph nor a $P_{4}$-free graph, so it is of interest to investigate if it is a graph $G$ with $\gamma(G)=\gamma_{[1,2]}(G)$.

By the definition of $[1,2]$-domination, it can be seen that

Lemma 1 For any graph $G, \gamma(G) \leq \gamma_{[1,2]}(G)$.

In (Klavžar \& Seifter, 1995), it was prove that

Theorem 8 (Klavžar \& Seifter, 1995) $\gamma\left(T_{3, n}\right)=n-\left\lfloor\frac{n}{4}\right\rfloor$ for $n \geq 4$.

Theorem 9 (Klavžar \& Seifter, 1995) $\gamma\left(T_{4, n}\right)=n$ for $n \geq 4$.

Theorem $10 \gamma_{[1,2]}\left(T_{3, n}\right)=n-\left\lfloor\frac{n}{4}\right\rfloor$ for $n \geq 4$.

Proof. The lower bounds follows from Lemma 1 and Theorem 8 . Now we give the upper bounds. 
Case 1. $n \in \mathbb{Z}_{4}^{0}$. Let

$$
P=\left[\begin{array}{llll}
0 & 0 & 1 & 0 \\
1 & 0 & 0 & 0 \\
0 & 0 & 1 & 0
\end{array}\right] .
$$

By repeating the pattern $P$ for $\frac{n}{4}$ times, we obtain a [1,2]-set of weight $\frac{3 n}{4}$. Therefore, $\gamma_{[1,2]}\left(T_{3, n}\right) \leq n-\left\lfloor\frac{n}{4}\right\rfloor$.

Case 2. $n \in \mathbb{Z}_{4}^{1}$.

Let

$$
P_{1}=\left[\begin{array}{llllll}
0 & 0 & 1 & 0 & \vdots & 0 \\
1 & 0 & 0 & 0 & \vdots & 1 \\
0 & 0 & 1 & 0 & \vdots & 0
\end{array}\right] .
$$

By repeating the leftmost 4 columns of the pattern $P_{1}$ for $\frac{n-1}{4}$ times, we obtain a [1,2]-set of weight $n-\left\lfloor\frac{n}{4}\right\rfloor$. Therefore, $\gamma_{[1,2]}\left(T_{3, n}\right) \leq n-\left\lfloor\frac{n}{4}\right\rfloor$

Case 3. $n \in \mathbb{Z}_{4}^{2}$.

Let

$$
P_{2}=\left[\begin{array}{lllllll}
0 & 0 & 1 & 0 & \vdots & 0 & 0 \\
1 & 0 & 0 & 0 & \vdots & 1 & 0 \\
0 & 0 & 1 & 0 & \vdots & 0 & 1
\end{array}\right] .
$$

By repeating the leftmost 4 columns of the pattern $P_{2}$ for $\frac{n-2}{4}$ times, we obtain a [1,2]-set of weight $n-\left\lfloor\frac{n}{4}\right\rfloor$. Therefore, $\gamma_{[1,2]}\left(T_{3, n}\right) \leq n-\left\lfloor\frac{n}{4}\right\rfloor$.

Case 4. $n \in \mathbb{Z}_{4}^{3}$.

Let

$$
P_{3}=\left[\begin{array}{llllllll}
0 & 0 & 1 & 0 & \vdots & 0 & 1 & 0 \\
1 & 0 & 0 & 0 & \vdots & 1 & 0 & 0 \\
0 & 0 & 1 & 0 & \vdots & 0 & 1 & 0
\end{array}\right] .
$$

By repeating the leftmost 4 columns of the pattern $P_{3}$ for $\frac{n-3}{4}$ times, we obtain a [1,2]-set of weight $n-\left\lfloor\frac{n}{4}\right\rfloor$. Therefore, $\gamma_{[1,2]}\left(T_{3, n}\right) \leq n-\left\lfloor\frac{n}{4}\right\rfloor$.

Theorem $11 \gamma_{[1,2]}\left(T_{4, n}\right)=n$ for $n \geq 4$. Proof. The lower bounds follows from Lemma 1 and Theorem 9. Now we give the upper bounds.

Case 1. $n \in \mathbb{Z}_{4}^{0}$.

Let

$$
P=\left[\begin{array}{llll}
0 & 0 & 1 & 1 \\
0 & 0 & 0 & 0 \\
1 & 1 & 0 & 0 \\
0 & 0 & 0 & 0
\end{array}\right] .
$$

By repeating the pattern $P$ for $\frac{n}{4}$ times, we obtain a [1,2]-set of weight $n$ of of $T_{4, n}$. Therefore, $\gamma_{[1,2]}\left(T_{4, n}\right) \leq n$.

Case 2. $n \in \mathbb{Z}_{4}^{1}$.

Let

$$
P_{1}=\left[\begin{array}{llllllllll}
0 & 0 & 1 & 1 & \vdots & 0 & 1 & 0 & 0 & 1 \\
0 & 0 & 0 & 0 & \vdots & 0 & 0 & 0 & 1 & 0 \\
1 & 1 & 0 & 0 & \vdots & 1 & 0 & 0 & 0 & 0 \\
0 & 0 & 0 & 0 & \vdots & 0 & 0 & 1 & 0 & 0
\end{array}\right] \text {, and } P_{1}^{\prime}=\left[\begin{array}{ccccc}
0 & 1 & 0 & 0 & 1 \\
0 & 0 & 1 & 0 & 0 \\
1 & 0 & 0 & 0 & 0 \\
0 & 0 & 0 & 1 & 0
\end{array}\right] \text {. }
$$

For $n \geq 9$, by repeating the leftmost 4 columns of the pattern $P_{1}$ for $\frac{n-1}{4}$ times, we obtain a [1,2]-set of weight $n$ of $T_{4, n}$. Therefore, $\gamma_{[1,2]}\left(T_{4, n}\right) \leq n$. The pattern $P_{1}^{\prime}$ induces a [1,2]-set of weight 5 of $T_{4,5}$. Therefore, $\gamma_{[1,2]}\left(T_{4, n}\right) \leq n$ for each $n \geq 5$ in this case. 
Case 3. $n \in \mathbb{Z}_{4}^{2}$.

Let

$$
P_{2}=\left[\begin{array}{lllllll}
0 & 0 & 1 & 1 & \vdots & 0 & 1 \\
0 & 0 & 0 & 0 & \vdots & 0 & 0 \\
1 & 1 & 0 & 0 & \vdots & 1 & 0 \\
0 & 0 & 0 & 0 & \vdots & 0 & 0
\end{array}\right] .
$$

By repeating the leftmost 4 columns of the pattern $P_{2}$ for $\frac{n-2}{4}$ times, we obtain a [1,2]-set of weight $n$ of $T_{4, n}$. Therefore, $\gamma_{[1,2]}\left(T_{4, n}\right) \leq n$.

Case 4. $n \in \mathbb{Z}_{4}^{3}$.

Let

$$
P_{3}=\left[\begin{array}{llllllll}
0 & 0 & 1 & 1 & \vdots & 0 & 1 & 1 \\
0 & 0 & 0 & 0 & \vdots & 0 & 0 & 0 \\
1 & 1 & 0 & 0 & \vdots & 1 & 0 & 0 \\
0 & 0 & 0 & 0 & \vdots & 0 & 0 & 0
\end{array}\right] .
$$

By repeating the leftmost 4 columns of the pattern $P_{3}$ for $\frac{n-3}{4}$ times, we obtain a [1,2]-set of weight $n$ of $T_{4, n}$. Therefore, $\gamma_{[1,2]}\left(T_{4, n}\right) \leq n$.

\section{Conclusion}

We show that the [2,b]-domination problem is NP-complete for $b$ at least 3 , and the [1,2]-total domination problem is NP-complete. Chellali et al. asked which graph satisfies the domination number equals to the $[1,2]$ domination number. We determine the [1,2]-total domination and [1,2] domination numbers of toroidal grids with three rows and four rows, and show that this class of graphs satisfied the above condition.

\section{Acknowledgements}

This work was supported by Applied Basic Research (Key Project) of Sichuan Province under grant 2017JY0095 , Key Project of Sichuan Provincial Department of Education under grant 17ZA0079 and Automotive Creative Design Pilot Area of Chengdu University and Longquanyi District under grant 2015-CX00-00010-ZF.

\section{References}

Bondy, J. A., \& Murty, U. S. R. (1976). Graph theory with applications. MACMILLAN.

Chellali, M., Haynes, T. W., Hedetniemi, S. T., \& McRae, A. (2013). [1, 2]-sets in graphs. Discrete Applied Mathematics, 161(18), 2885-2893. http://dx.doi.org/10.1016/j.dam.2013.06.012

Dreyer Jr, P. A. (2000). Applications and variations of domination in graphs. Doctoral dissertation, Rutgers, The State University of New Jersey.

Fiala, J., Golovach, P. A., Kratochvíl, J., Lidický, B., \& Paulusma, D. (2012). Distance three labelings of trees. Discrete Applied Mathematics, 160(6), 764-779. http://dx.doi.org/10.1016/j.dam.2011.02.004

Goharshady, A. K., Hooshmandasl, M. R., \& Meybodi, M. A. (2016). [1, 2]-sets and [1, 2]-total sets in trees with algorithms. Discrete Applied Mathematics, 198, 136-146. http://dx.doi.org/10.1016/j.dam.2015.06.014

Hammack, R. H., Imrich, W., Klavžar, S. (2011). Handbook of product graphs. Boca Raton: CRC press.

Klavžar, S., \& Seifter, N. (1995). Dominating Cartesian products of cycles. Discrete Applied Mathematics, 59(2), 129136. http://dx.doi.org/10.1016/0166-218X(93)E0167-W

Schaefer, T. J. (1978). The complexity of satisfiability problems. In Proceedings of the tenth annual ACM symposium on Theory of computing, 216-226. ACM. http://dx.doi.org/10.1145/800133.804350

Thiagarajan, M., \& Bhaskaram, R. (2015). Total Domination Number of Product of Cycles. International Journal of Mathematical Sciences \& Applications, 3(2),121-127.

Yang, X., \& Wu, B. (2014). [1, 2]-domination in graphs. Discrete Applied Mathematics, 175, 79-86.

http://dx.doi.org/10.1016/j.dam.2014.05.035 


\section{Copyrights}

Copyright for this article is retained by the author(s), with first publication rights granted to the journal.

This is an open-access article distributed under the terms and conditions of the Creative Commons Attribution license (http://creativecommons.org/licenses/by/4.0/). 